\title{
Analysis of Player Statistics in Major League Baseball Players before and after Achilles Tendon Repair
}

\author{
Deniz Can Ince
}

Received: 16 October 2017/Accepted: 8 December 2017/Published online: 27 February 2018

(C) Hospital for Special Surgery 2018

Dear Editor,

I read the study by Bryan M. Saltzman et al. entitled "Analysis of Player Statistics in Major League Baseball Players Before and After Achilles Tendon Repair" with great interest [1]. I would like to congratulate the authors for a detailed and valuable study. However, I felt there are some aspects the authors could clarify.

First, I am wondering if the authors considered the age of players, the years of Achilles tendon tears, and the proper aging curve before claiming a significant change in strikeouts. The study's reported 5\% increase in strikeouts between nonpower-side Achilles tears and power-side Achilles tears may be a result of the upward trend of strikeouts in Major League Baseball (MLB) and the natural decrease of productivity as players age, as opposed to a significant decrease in player performance due to the injury's laterality. In recent years, the strikeout rate $(\mathrm{K} \%)$ has increased. By my calculation, data from the same sources that the authors used, www.fangraphs.com and www.baseball-reference.com, indicate that $\mathrm{K} \%$ between 2013 and 2008 is $2.02 \%$ higher than between 2005 and 1998 [2]. Additionally, because the mean age reported in the study is $31.6 \pm 3.2$ years, an aging curve must be considered. FanGraphs' K\% aging curve shows that players between the ages of 31 and 32 years from 2013 through 2006 had nearly a $3 \%$ increase in strikeouts compared to the league average over those years $(18.3 \%)$, while players between the ages of 31 and 32 years from the 2005 through the 1998 seasons had less than a $1 \%$ increase in strikeouts compared to the league average over those years (16.7\%) [3]. Therefore, a 31-year-old who tears his Achilles tendon could expect a $\mathrm{K} \%$ variance of more than $3 \%$ only based on what year the injury occurred. Therefore, age, year of injury, and

\footnotetext{
Electronic supplementary material The online version of this article (https://doi.org/10.1007/s11420-017-9602-4) contains supplementary material, which is available to authorized users.

D. C. Ince, $(\bowtie)$

University Scholars in Medicine, Washington University,

St. Louis, MO, USA

e-mail: dcince@wustl.edu
}

proper aging curve must be presented and applied before a significant change can be claimed.

Second, how can the authors justify the players' speeds as "detrimentally affected" when the study's ultimate baserunning statistic shows no significant correlation among Achilles tears compared to the control or to the power/nonpower side of the tear? The study reports that the significant decrease in speed score (a statistic that attempts to demonstrate a player's ability on the base paths) based on the injury's laterality suggests "that the careers of those with Achilles tendon rupture to the power-generating rear leg are detrimentally affected." This statement could be misleading, as speed score has become relatively obsolete because it only uses four data points. Ultimate base running has become a more prevalent metric than speed score because it incorporates more data points, and this more detailed statistic shows that there is no significant difference in the players' ability on the base paths.

\section{Compliance with Ethical Standards}

Conflict of Interest: Deniz Can Ince declares that he has no conflicts of interest.

Human/Animal Rights: N/A.

Informed Consent: N/A.

Required Author Forms Disclosure forms provided by the author are available with the online version of this article.

\section{References}

1. Saltzman BM, Tetreault MW, Bohl DD, Tetreault D, Lee S, Bach BR. Analysis of player statistics in Major League Baseball players before and after Achilles tendon repair. HSS J. 2017;13(2): 108-118.

2. Fangraphs.com. League stats. Available at http://www.fangraphs. $\operatorname{com} /$ leaders. aspx ? pos $=$ all $\&$ stats $=$ bat $\& 1 \mathrm{~g}=\mathrm{a} 11 \&$ qual $=0 \&$ type $=8 \&$ season $=2016 \&$ month $=0 \&$ seas on $1=1871 \&$ ind $=0 \&$ team $=0$,ss\&rost $=0 \&$ age $=0 \&$ filter $=\&$ players $=0 \&$ sort $=1, \mathrm{~d}$

3. Fangraphs.com. Component changes in new hitter aging curves. Available at http://www.fangraphs.com/blogs/component-changes-in-new-hitter-aging-curves 